\title{
Dynamics of strong-field photoionization in two dimensions for short-range binding potentials
}

\author{
J. Matulewski; A. Raczyński and J. Zaremba \\ Instytut Fizyki,Uniwersytet Mikołaja Kopernika, \\ ul. Grudziądzka 5, 87-100 Toruń, Poland
}

November 13, 2018

\begin{abstract}
Photodetachment in an ultrastrong laser field in two spatial dimensions is investigated $a b$ initio. A qualitative behavior of the packet for a short-range binding potential is contrasted with that for a soft-core potential, in particular dynamical effects due to a rescattering of fragments separated from the main packet are demonstrated.
\end{abstract}

\section{Introduction}

The problem of ultrastrong-field photoionization has been the subject of studies in quantum optics for the last fifteen years (for a recent review see Ref. [1]). Because of enormous technical difficulties most of ab initio calculations were performed for one dimensional models. It is well known that if the electric field amplitude $\varepsilon_{0}$ grows in the region of a few atomic units (for a typical frequency $\omega=1$ a.u. and a binding potential supporting an initial state of energy of order of 1 a.u.), the ionization rate need not grow monotonically

*e-mail: jacek@phys.uni.torun.pl 
with the field and the population can be regarded as trapped in a KramersHenneberger (KH) well [2]. At a field amplitude of about 10 a.u. almost no probability leaks from the neighborhood of the nucleus. Unfortunately, one-dimensional models fail to represent important features of real systems; in particular they can imitate only an interaction with a linearly polarized field in the dipole approximation. Thus investigating magnetic interactions or light polarization effects requires a generalization to models with more spatial dimensions [3]. Investigations performed in two dimensions for longrange binding potentials predict in particular that the stabilization at field intensities higher than $10 \mathrm{a} . \mathrm{u}$. is destroyed by a nondipole kinetic effect the so-called magnetic drift of the packet as a whole away from the region of the nucleus.

The aim of this paper is to study photodetachment in superintense fields (such that the Schrödinger equation is still valid) in two dimensions in the situation which has not yet been investigated in a few so far existing papers $[3,4,5]$; in comparison with other papers the long-range atomic potential is replaced by a short-range potential well (rectangular). The details of the dynamics in our case include some special effects, an interpretation of which is necessary for a complete understanding of the process.

A new element in comparison with earlier papers [3] is that the well potential (especially if it has deep eigenstates), due to its steep edges, causes tearing of the oscillating wave packet into fragments, even in such strong laser field intensities as 15 - 20 a.u. Because of the wavepacket tearing, the classical model does not allow to satisfactorily interpret all numerical results. In particular satellite wavepacket's fragments detached at different times and being of a significant size can be rescattered in their oscillatory motion and interfere, with the details of the process depending on the shape of the laser pulse, in particular on the position of the classical packet's turning points. The phase dependence of the dynamics is also discussed. 


\section{The binding potential and the shape of the pulse}

There are two types of model atomic potentials commonly used in the studies on the interaction of atomic systems with superstrong fields in one-dimension: a soft core Coulomb potential proposed by Su, Javanainen and Eberly in a series of papers [4] and a short-range potential of a negative ion, modeled, e.g., by a rectangular well. The main difference is that the former potential is smooth and supports an infinite number of discrete states while the latter one has two points of discontinuity and a few bound states. In the latter case the packet has less chances to remain a connected structure (i.e. it is more exposed to tearing) and cannot be dynamically stabilized, i.e. trapped in the series of the Rydberg states.

While two-dimensional studies on the strong-field stabilization of models with a soft core potential have already been reported (see [1], [6]), in this paper we concentrate on special effects due to a potential well, which we assume to be radial, i.e. we take

$$
V(\vec{r})=-V_{0} \theta(a-r),
$$

where $r=\sqrt{x^{2}+y^{2}}$. The width $a$ and the depth $V_{0}$ of the well are chosen so that there is only a single (initial) bound state.

The choice of the pulse shape is also very important for the dynamics of the ionization process. The stabilization is possible only if the pulse does not lead to a fast drift of the electron, i.e. the classical trajectory of the electron in the absence of the binding potential remains close to the nucleus. The results presented below are obtained for rectangular cosinusoidal pulses which satisfy this condition. For a pulse propagating along the $y$ axis and with $x$ polarization we thus take the field amplitude $\vec{\varepsilon}(y, t)=\hat{x} \varepsilon_{0} \cos (k y-\omega t)$

or the vector potential $\vec{A}(y, t)=-\hat{x} \frac{\varepsilon_{0}}{\omega} \sin (k y-\omega t)$. The continuous pulses often adopted in other papers (e.g. those with trapezoid envelope) may imply complicated switch-on effects, especially if no dipole approximation is made. For example they may give raise to a field component which is not an electromagnetic wave. Moreover, calculating the vector potential by a time integral of the electric field introduces ambiguities reflecting various possible experimental realizations. We have checked that the effect we describe below 
appears also in a modified version for a trapezoid envelope.

The cosine pulse with any initial phase has an important feature in contradistinction to other pulses usually assumed in this context, namely the turning points are shifted asymmetrically with respect to the potential well, with one of them located at the well, i.e. at the initial position of the wave packet. If $\varepsilon(t)=\varepsilon_{0} \cos (\omega t)$, the electron free of the binding potential would, in one dimension, oscillate in the range $\left(-2 \varepsilon_{0} / \omega^{2}, 0\right)$, which means that the right turning point occurs at the well.

Strong field ionization is very often described in terms of the KramersHenneberger $(\mathrm{KH})$ well. In Kramers frame an unbounded electron stays at rest and experiences the interaction of the oscillating nucleus. The $\mathrm{KH}$ potential well is just this interaction averaged over the oscillation period or, in other words, the zero-th term of the Fourier series of the interaction. In the initial stage of ionization the electron's wavepacket spreads out until it has reached the size of the KH well. Further changes of the packet's shape as well as its slow drift may be explained as due to higher terms of the Fourier expansion.

For the cosinusoidal pulse the electron is initially located close to one of the two minima of the $\mathrm{KH}$ well, in contradistinction to the case of other pulses studied in this context, for which its initial position occurs in the middle.

In the present work we will demonstrate how the results of one-dimensional computations must be completed and developed in the case of two spatial dimensions. In particular the Kramers frame and the corresponding $\mathrm{KH}$ well are now introduced as if there were no movement in the direction perpendicular to the electric field $\vec{\varepsilon}(\vec{r}, t)=\hat{x} \varepsilon_{0} \cos (k y-\omega t)$ : the transformation accounts for the electron movement $X(t)=\frac{\varepsilon_{0}}{\omega^{2}}(1-\cos (\omega t))$, while its slow drift in the $x$ direction and/or its throwing away of small parts in both directions are due to the higher terms of the Fourier expansion. The evolution in the $y$ direction includes also magnetic effects seen as throwing away of small pieces rather than a drift of the packet as a whole. This new aspect of the two-dimensional ionization process has not been observed in other works on strong-field ionization because they were dealing with soft-core binding potentials, in contradistinction to short-range potentials considered in this paper. 
The KH well has the form

$$
V_{K H}\left(x, y ; \varepsilon_{0}\right)=\frac{1}{T} \int_{t}^{t+T} V(x-X(\tau), y) d \tau,
$$

where $T=2 \pi / \omega$. The analytical form of the above $\mathrm{KH}$ well is

$$
V_{K H}\left(x, y ; \varepsilon_{0}\right)=-\frac{V_{0}}{\pi} \Theta\left(r^{2}-y^{2}\right)\left(\arcsin \left(\alpha_{+}\right)-\arcsin \left(\alpha_{-}\right)\right),
$$

where

$$
\alpha_{ \pm}= \begin{cases}1, & x< \pm \sqrt{a^{2}-y^{2}} \\ 1 \pm \frac{\omega^{2}}{\varepsilon_{0}}\left(\sqrt{a^{2}-y^{2}} \mp x\right) & x>2 \pm \sqrt{a^{2}-y^{2}} \\ -1, & x\end{cases}
$$

The KH potential for $\varepsilon_{0}=15$ a.u., $\omega=1$ a.u. and for the radial well (Eq. (2) with $a=1$ a.u., $V_{0}=2$ a.u., a single bound state) and its two eigenstates are shown in Fig. 1.

\section{Quantum numerical results of ab initio sim- ulations and classical predictions}

Because we want to include nondipole effects we write the atom-field interaction in the velocity gauge. The vector potential $\hat{x} A(k y-\omega t)$ is polarized along $x$ axis and the Hamiltonian reads

$$
\hat{H}(\vec{r}, t)=\frac{(\hat{p}+\hat{x} A(k y-\omega t))^{2}}{2}+V(\vec{r}),
$$

where the radial potential is given by Eq. (2).

We have solved this equation both with and without the dipole approximation using the alternating direction implicit method (ADI) [7], which allows one to use tridiagonal sets of equations which can be solved easily and fast. The main difficulty in the two-dimensional calculation is not the number of operations to be performed by a processor but rather a large memory necessary to store the results. The size of the accessible memory sets a limit 
to the size of the spatial grid used in the calculation. We performed our calculation on the $2048 \times 2048$ grid with the step $\Delta x$ close to 0.1 a.u. The space range is from -100 a.u. to +100 a.u. in both directions.

As mentioned above the particular form of the vector potential was

$$
\vec{A}(y, t)=-\hat{x} \frac{\varepsilon_{0}}{\omega} \sin (k y-\omega t)
$$

with $\omega=1$ a.u. and $\varepsilon_{0}$ equal to 15 and 20 a.u. Larger values of the vector potential amplitude require a relativistic approach.

At the beginning of each cycle the left minimum of the $\mathrm{KH}$ well occurs close to the packet's initial position in the well; in fact Eq. (3) yields a shift of the maximum of the wavepacket trapped by the $\mathrm{KH}$ well with respect to the initial wavepacket's maximum by about $1 \mathrm{a.u}$. The latter is localized in the KH well's left half and is almost equally divided between the two discrete states. The energy difference between the $\mathrm{KH}$ eigenstates is small, so we do not observe any beats, which would occur in a much longer time scale than that considered here. The shift of the minima of the two wells is reflected on the dynamics of the initial state population (see Fig. 2). The splitting of each peak can be explained by the fact that the maximum of the trapped packet passes by the original well twice at the beginning of each period, with small velocities of opposite signs. This can be treated as an evidence of applicability of $\mathrm{KH}$ approach in this range of parameters.

The rest of the initial wavepacket, i.e. the part which has not been trapped in the KH well as well as the part initially trapped but later released due to higher terms of the Fourier expansion, is thrown away in the form of concentric rings (see Fig. 3) which oscillate as a whole along the $x$ axis in the rhythm of the field. In the dipole approximation neither turn of the $y$ axis is distinguished but if no dipole approximation is made most of probability will drift towards the positive direction of the $y$ axis due to the magnetic force, according to the classical considerations presented below. The additional structures visible in Fig. 3 will be discussed later.

The vector potential given by Eq. (6) corresponds to the electric and magnetic fields: 


$$
\begin{gathered}
\vec{E}(y, t)=\hat{x} E(y, t)=\hat{x} \varepsilon_{0} \cos (k y-\omega t), \\
\vec{B}(y, t)=-\hat{z} B(y, t)=-\hat{z} B_{0} \cos (k y-\omega t), \quad \text { where } \quad \vec{k}=\hat{y} k=\hat{y} \frac{y}{c} .
\end{gathered}
$$

Then the Lorentz force is

$$
\vec{F}=-(\vec{E}+\vec{v} \times \vec{B})=-\left[E-v_{y} B, v_{x} B, 0\right]
$$

One can write the classical equations of motion for an electron in the electromagnetic field

$$
\left\{\begin{array}{l}
\ddot{x}(t)=-\varepsilon_{0}\left(1-\frac{\dot{y}(t)}{c}\right) \cos (k y(t)-\omega t) \\
\ddot{y}(t)=-\frac{\varepsilon_{0}}{c} \dot{x}(t) \cos (k y(t)-\omega t)
\end{array}\right.
$$

which can be easily solved numerically. The solution of these equations is presented in Fig. 4. The upper plot shows fast oscillations with the frequency $\omega$ and the same amplitude as for the corresponding one-dimensional solution in the dipole approximation. The lower plot shows the magnetic drift and the oscillations of the frequency $2 \omega$. The velocity of this drift is proportional to the square of electric field amplitude $\varepsilon_{0}$. The results can be easily understood if we simplify equations (10) neglecting the magnetic component of the Lorentz force in the $x$ direction and neglecting at this stage the $y$ dependence of the field. So we can write the simplified equations of motion

$$
\left\{\begin{array}{l}
\ddot{x}(t)=-\varepsilon_{0} \cos (\omega t), \dot{x}(t)=-\frac{\varepsilon_{0}}{\omega} \sin (\omega t), \\
\ddot{y}(t)=-\frac{\varepsilon_{0}}{c} \dot{x}(t) \cos (\omega t)=\frac{\varepsilon_{0}^{2}}{2 \omega c} \sin (2 \omega t),
\end{array}\right.
$$

with the electron initially resting at $x=y=0$.

This approximation corresponds to the series expansion of vector potential $A_{0} \sin (k y-\omega t)$ around $\omega t$ and leaving only the lowest term. The magnetic drift is directed towards the positive values of $y$, with oscillations of frequency $2 \omega$ imposed on it. The average velocity in the $y$ direction is proportional to the square of the field amplitude. 


\section{Wavepacket tearing and its consequences}

The ab initio solutions of the Schrödinger equation with the Hamiltonian (5) and the laser field defined by (6) demonstrate that the mean values of the operators $x$ and $y$ (see Fig. 5) look very similar to the classical predictions (Fig. 4), as could be expected from the Ehrenfest theorem.

The wavefunctions (see Figs 3, 6 and 7) look quite different from those shown in Refs [6] and [4], because here a deep potential well is used instead of a soft-core atom. In spite of the strength of the laser field, the wave function does not behave typically for the over-the barrier (OTB) ionization regime, in which the packet behaves essentially as a connected structure. Parts of the packet are thrown away in the form of two sets of elliptic rings with centers corresponding to the positions of both minima of the $\mathrm{KH}$ well. Those oval structures carry a significant portion of the probability of finding the electron. In the dipole approximation a new ring appears once a period (Fig. 7). If no dipole approximation is made, the rings appear twice a period with each second ring being due only to the magnetic force oscillating with the doubled frequency (cf. Eq. (11)) - see the inset of Fig. 6. The torn-off structures are thrown preferably towards positive $y$ due to the magnetic interaction. A comparison of the heights of the main peak in Figs 6 and 7 shows that making the dipole approximation leads to a significant underestimation of the ionization rate.

In Fig. 5, similarly as in Fig. 4, we can see fast oscillations in the $x$ direction in the upper plot and the magnetic drift in the $y$ direction in lower one. A slow drift, similar to that obtained in [8], is also visible at the graph of $\langle x\rangle$.

\section{Rescattering of the liberated parts of the wavepacket}

For the cosine rectangular pulse the wavepacket oscillates from 0 to $-2 \varepsilon_{0} / \omega^{2}$ along the $x$-axis like in the OTB regime. It is well known that an irreversible ionization of the packet in the form consisting in throwing away pieces of the packet can occur only if both the electric field and the binding potential are present and the packet has a small velocity when passing by the well. 
Those conditions are satisfied during a significant part of each cycle if the phase of the field is chosen so that the pulse is cosinusoidal. Then one of the wavepacket's turning points is located at the well so once a cycle an electron has the possibility to be liberated. In the case of a sine pulse with a trapezoid envelope (not shown here) there is no opportunity for any packet tearing in such strong laser fields until the wavepacket is wide enough to cover the range of classical oscillations (i.e. the width of $\mathrm{KH}$ well). In this case rings appear a few cycles later then in the case of cosine pulse.

As described above, for a cosine pulse at the end of each cycle, when the packet is stopping above the well, a part of the packet is thrown away from the well and forms a ring around the main part of the packet. Thus the wavefunction takes the shape of rings around the main maximum, oscillating as a whole in the $x$ direction. After a few periods the first oval structure reaches a radius of order of $2 \varepsilon_{0} / \omega^{2}$, so there is some probability of finding the electron at the well at the moment at which the classical turning point occurs at $-2 \varepsilon_{0} / \omega^{2}$, i.e. after an odd multiple of half a cycle. Tearing this secondary cloud causes a creation of a second family of rings, which are centered at the distance $2 \varepsilon_{0} / \omega^{2}$ from the center of the first family of rings and obviously carry a smaller portion of the probability.

The most interesting effect is an interference of both families of the rings giving rise to an additional structure between two turning points and to a complicated shape of the wavefunction at larger $x$ (see Fig. 3 (the wavefunction after $10 T$ computed in dipole approximation) and Figs 6 and 7). This interference effect has no magnetic character and occurs similarly both in the general case and in the dipole approximation.

This new type of rescattering can be even easier understood using the $\mathrm{KH}$ model. In the Kramers frame, i.e. in the frame oscillating like an unbound electron, the two wavepacket's turning points correspond to the two turning points of moving potential and, after time averaging, approximately to the minima of the KH well (for cosine pulse: $x=0$ and $x=+2 \varepsilon_{0} / \omega^{2}$ ). Because for the field magnitude used in this paper the $\mathrm{KH}$ well has two states with close energies (see Fig. 1) the electron is located in such a superposition of those states that only the left minimum is significantly populated. The higher terms of the Fourier expansion, characterized by large frequencies but rather small amplitudes, cause an ionization of the trapped wavepacket in a way typical of the multiphoton regime: fragments of the wavepacket are torn 
off and sent in all directions. It is how the first, main family of the rings in Figs 3 is formed. The torn-off pieces, being of a considerable size, are scattered on the other minimum of the $\mathrm{KH}$ well, which results in a formation of the second family of rings, centered about $2 \varepsilon_{0} / \omega^{2}$. Both families of the rings can interfere. In particular this is visible for the parts moving along the $x$-axis: a pattern structure is built after a few cycles between the two minima of the KH well.

In the case of a sine pulse with a trapezoid envelope the mechanism of tearing is similar as described above. An important difference is that it takes a few cycles for the packet to become broad enough so that it reaches the minima of the KH well and can be ionized by higher terms of the Fourier expansion. After that period the packet is rescattered on both minima of the $\mathrm{KH}$ well. Both families of rings appear simultaneously.

Our observations in two dimension allowed us to complete the interpretation of one dimensional results: a very similar interference structure in the form of numerous subpeaks between the classical turning points can also be seen in the results of one-dimensional computations (see Fig. 8). The effect

is significantly reduced if the well is made more shallow or if it is replaced by a soft-core potential.

\section{Conclusions}

We have presented a detailed dynamical picture of two-dimensional photodetachment in very strong fields in the parameters range not explored before. Due to the rapidly varying binding potential, for some model pulses one can observe tearing smaller pieces off the main packet. The latter pieces can themselves be torn into fragments, giving rise to new spatial structures of the wavepacket, possibly interfering with the old ones. We have demonstrated those elements of the dynamics that are due to the binding potential being short-range. The behavior of the packet described above is to be contrasted with the packet's dynamics in the case of a smooth binding potential, in which a connected structure is trapped in $\mathrm{KH}$ well and possibly push out by the magnetic force. 


\section{References}

[1] M. Gavrila, J. Phys B: At. Mol. Opt. Phys. 35 (2002) R147-R193

[2] for example J.H. Eberly, R. Grobe, C.K. Law, Q. Saw in Atoms in Intense Laser Fields, edited by M. Gavrila (Academic Press, Boston, 1992), p. 301

[3] Ionization in arbitrary polarization: see, e.g., M. Protopapas, D.G. Lappas and P.L. Knight Phys. Rev. Lett., 794550 (1997); the breakdown of the dipole approximation and magnetic drift: J.R. Vazquez de Aldana, L.Roso, Phys. Rev. A, 61, 043403 (2000), H.R. Reiss, Phys. Rev. A, 63, 013409

[4] for example J.H. Eberly, Q. Su, Phys. Rev. A 44 (1991) 5997

[5] M. Yu. Ryabikin and A. M. Sergeev, Opt. Express, 7, 417-426 (2000)

[6] J. R. Vazquez de Aldana, N.J. Kylstra, L. Roso, P.L. Knight, A. Patel, R.A. Worthington, Phys. Rev. A, 64, 013411 (2001)

[7] W.H. Press S.A.Teukolsky, W.T. Vetterling, B.P. Flannery, Numerical Recipes (Cambridge University Press 1992)

[8] J. Matulewski, A. Raczyński, J. Zaremba, Phys. Rev. A 61 (2000) 043402 
Figure 1: The KH averaged potential for a quantum well potential ( $a=1$ a.u., $V_{0}=2$ a.u.) and a cosine pulse $\left(\varepsilon_{0}=15\right.$ a.u., $\omega=1$ a.u. $)$. On the left the $\mathrm{KH}$ potential and the ground state wavefunction with energy $E_{1}=-0.0192$ a.u. On the right the wavefunction of the only excited state $\left(E_{2}=-0.0158\right.$ a.u. $)$.

Figure 2: The population of the initial state. The pulse parameters are $\varepsilon_{0}=15$ a.u., $\omega=1$ a.u. and the well potential $a=1$ a.u., $V_{0}=2$ a.u.

Figure 3: The modulus square of the wavefunction after $1 T, 2 T, 3 T, 4 T$, $4.8 T, 5 T, 10 T$ without the dipole approximation and after $10 T$ in the dipole approximation. The distance between the centers in the $x$ direction (horizontal) of the families of the rings is $30 \mathrm{a} . \mathrm{u}$. The atomic and field parameters are the same as in Fig. 2.

Figure 4: The solution of the Newton equations for an unbound electron without the dipole approximation $(x(t)$ on the upper plot and $y(t)$ on the lower one). The trajectory in the $x$ direction in the dipole approximation coincides with that without this approximation in the scale of the picture. A cosine pulse is assumed, with the intensity $\varepsilon_{0}=15$ a.u. and frequency $\omega=1$ a.u. One can see fast oscillations in the $x$ direction and the magnetic drift in the $y$ direction.

Figure 5: The mean values of $x$ and $y$ in the quantum simulation for the pulse parameters as in Fig. 4. One can see the slow drift in the $x$ direction and the magnetic drift in the $y$ direction.

Figure 6: Modulus square of the wavefunction after 6T. One can distinguish fragments thrown away after full cycles from those thrown away in the middle of each cycle (see inset). The parameters of a rectangular cosinusoidal pulse are $\varepsilon_{0}=20$ a.u., $\omega=1$ a.u. The shift of the probability density towards the region of $y>0$ is well visible.

Figure 7: As in Fig. 6 but in the dipole approximation. 
Figure 8: The main picture: a one-dimensional wavepacket for a rectangular pulse with the parameters as in Fig. 3 and the well potential chosen to match the energy of only state $E_{1}=-0.832$ a.u. The small pictures: upper wavepacket, the same field but for a soft-core atom [4]; lower wavepacket, the well potential four times less deep than in the main picture (the maxima on the small pictures have the height about 0.0004 a.u.). 


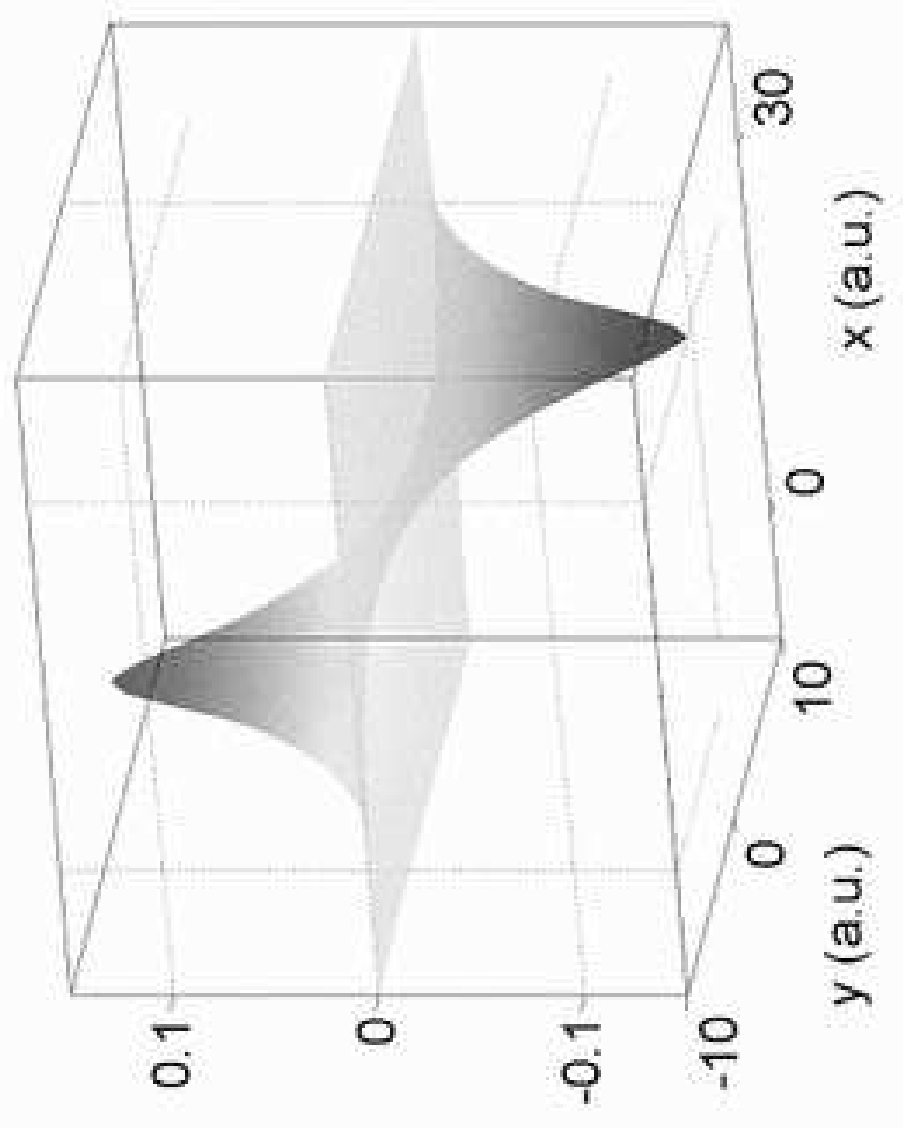

uo!̣ounฺฺәлем

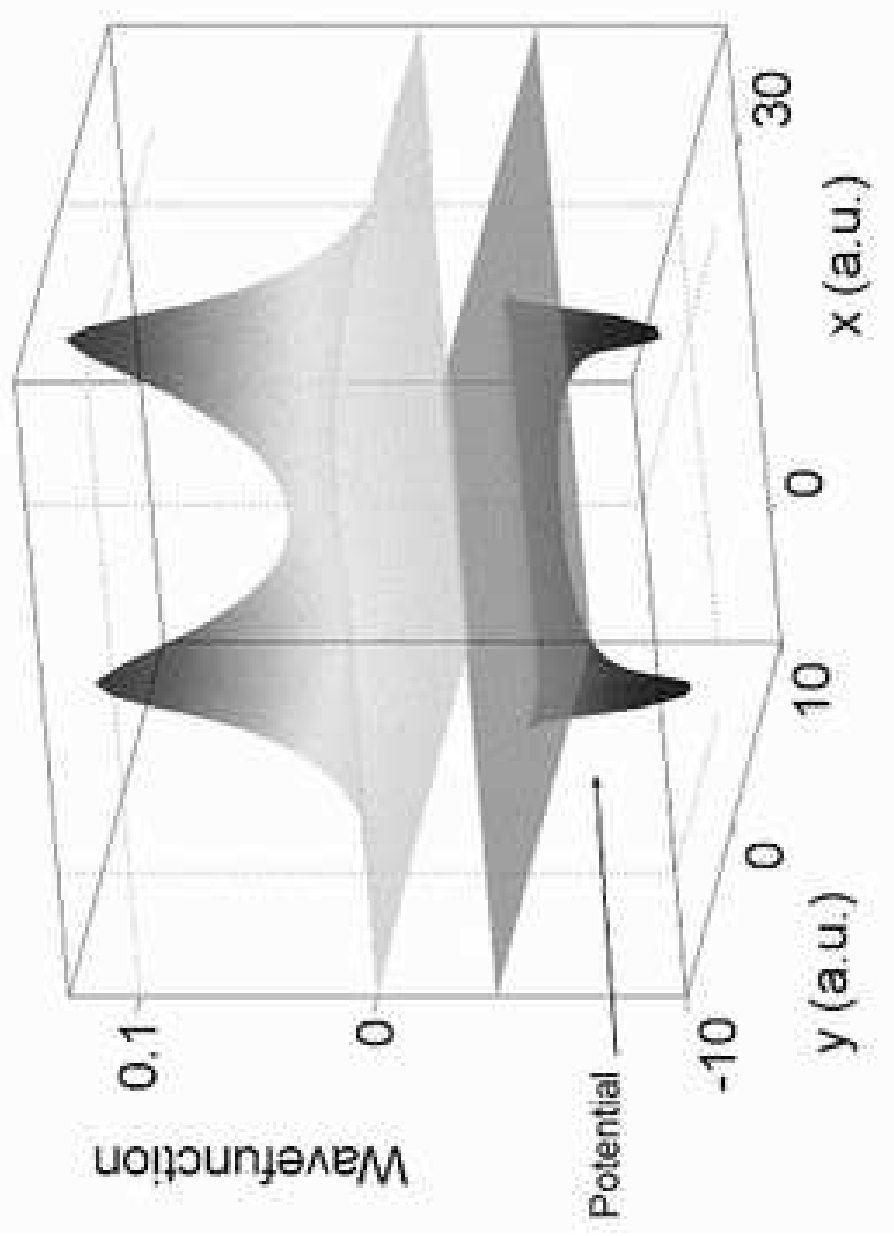




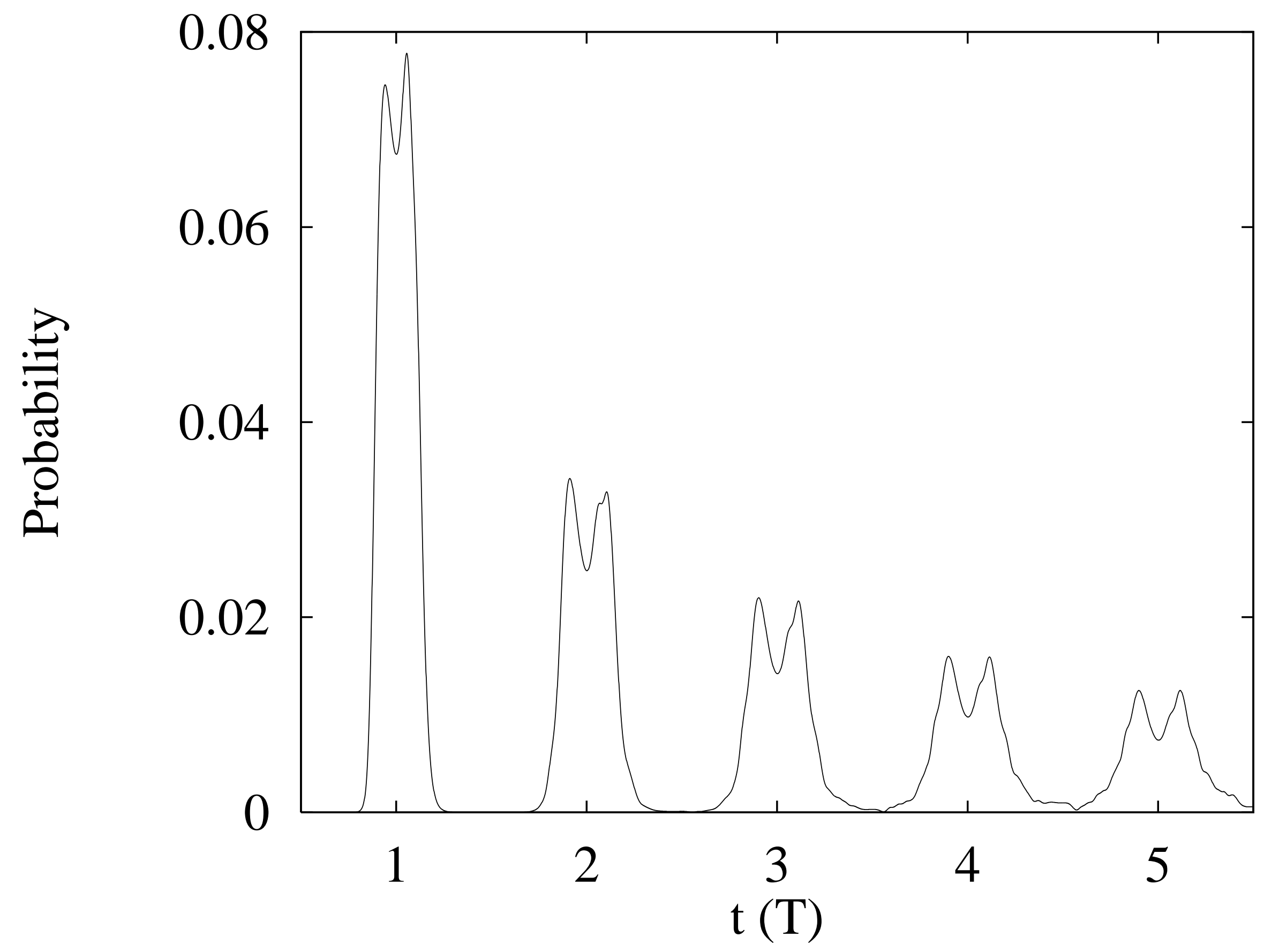




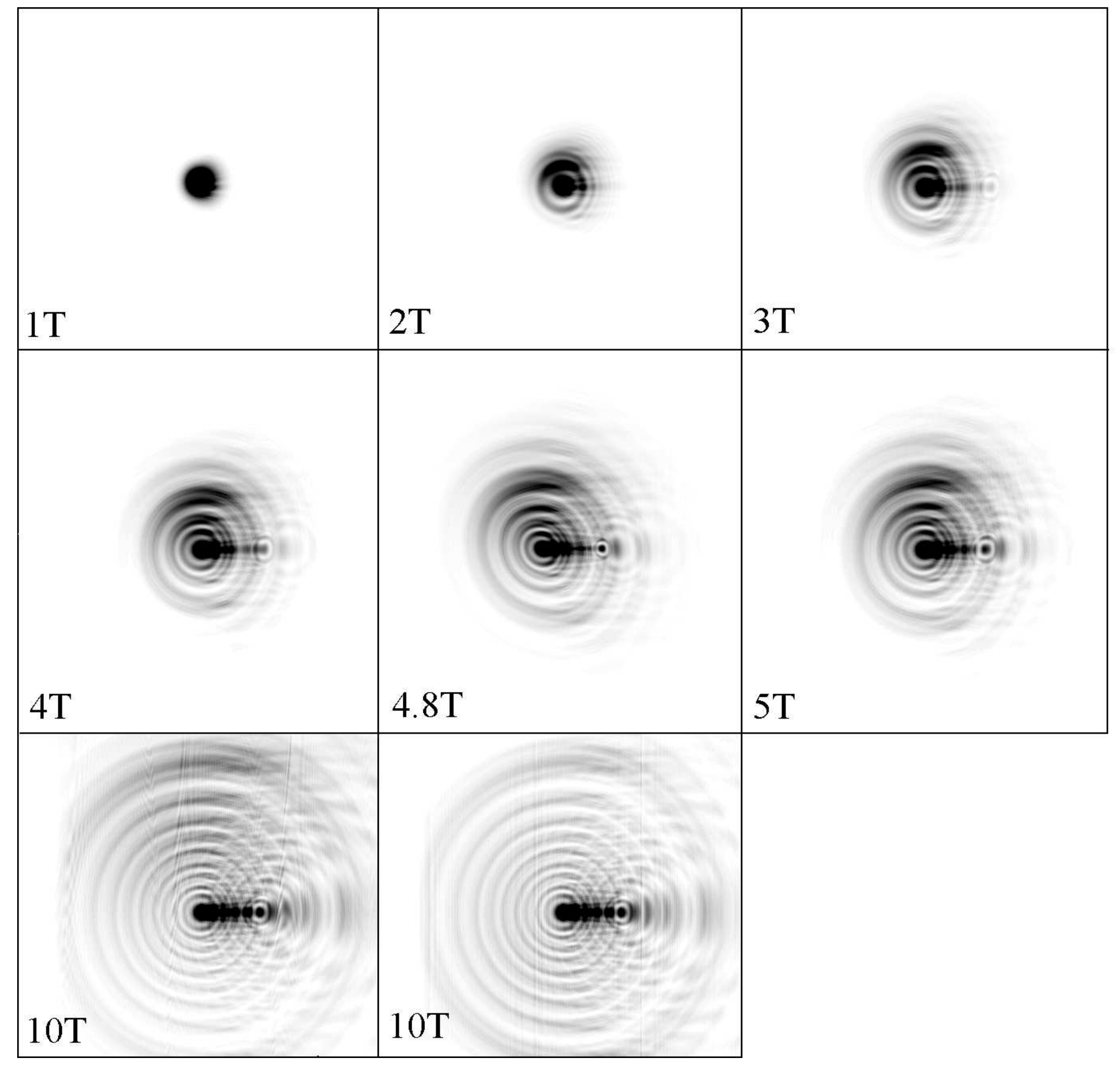




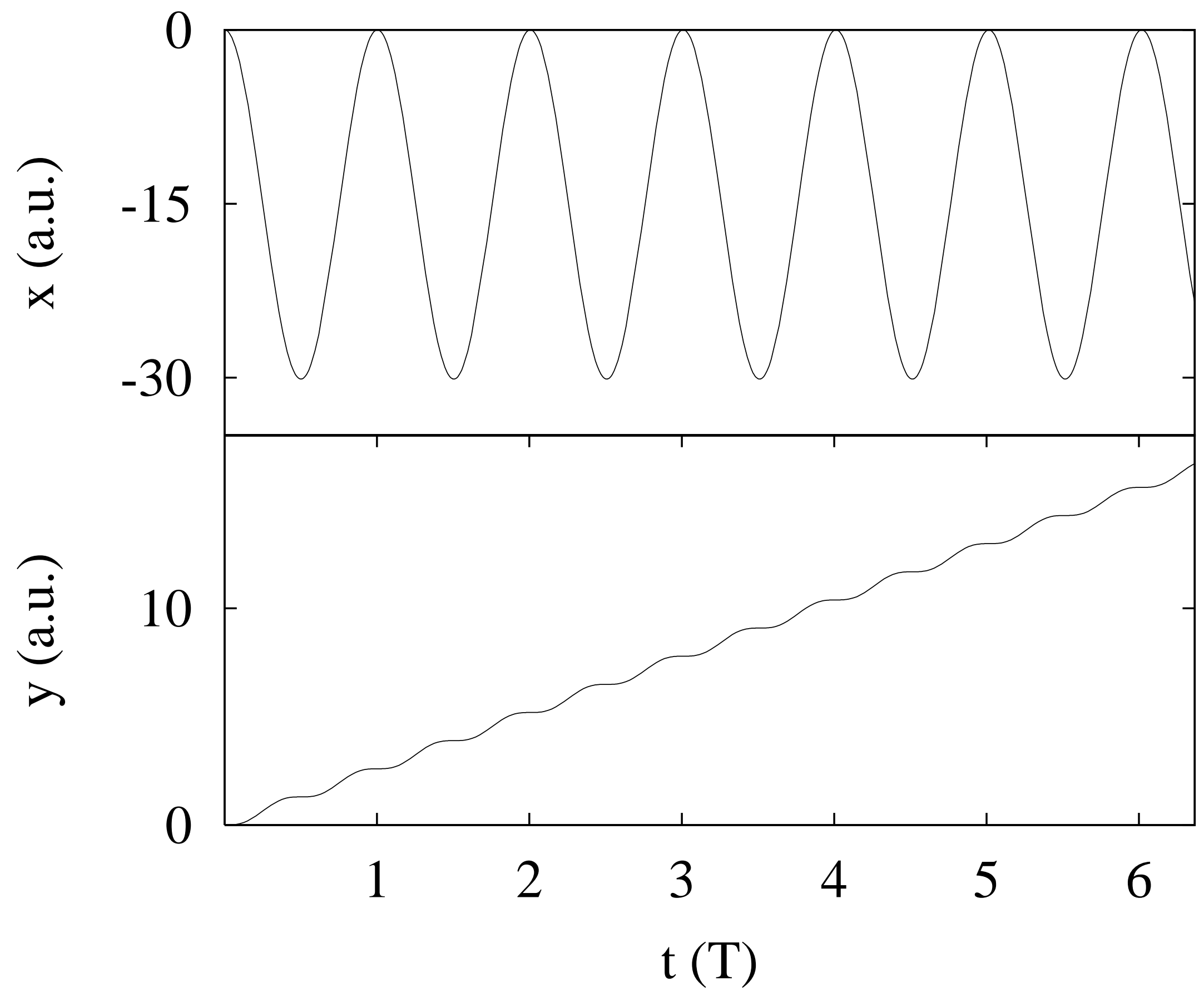




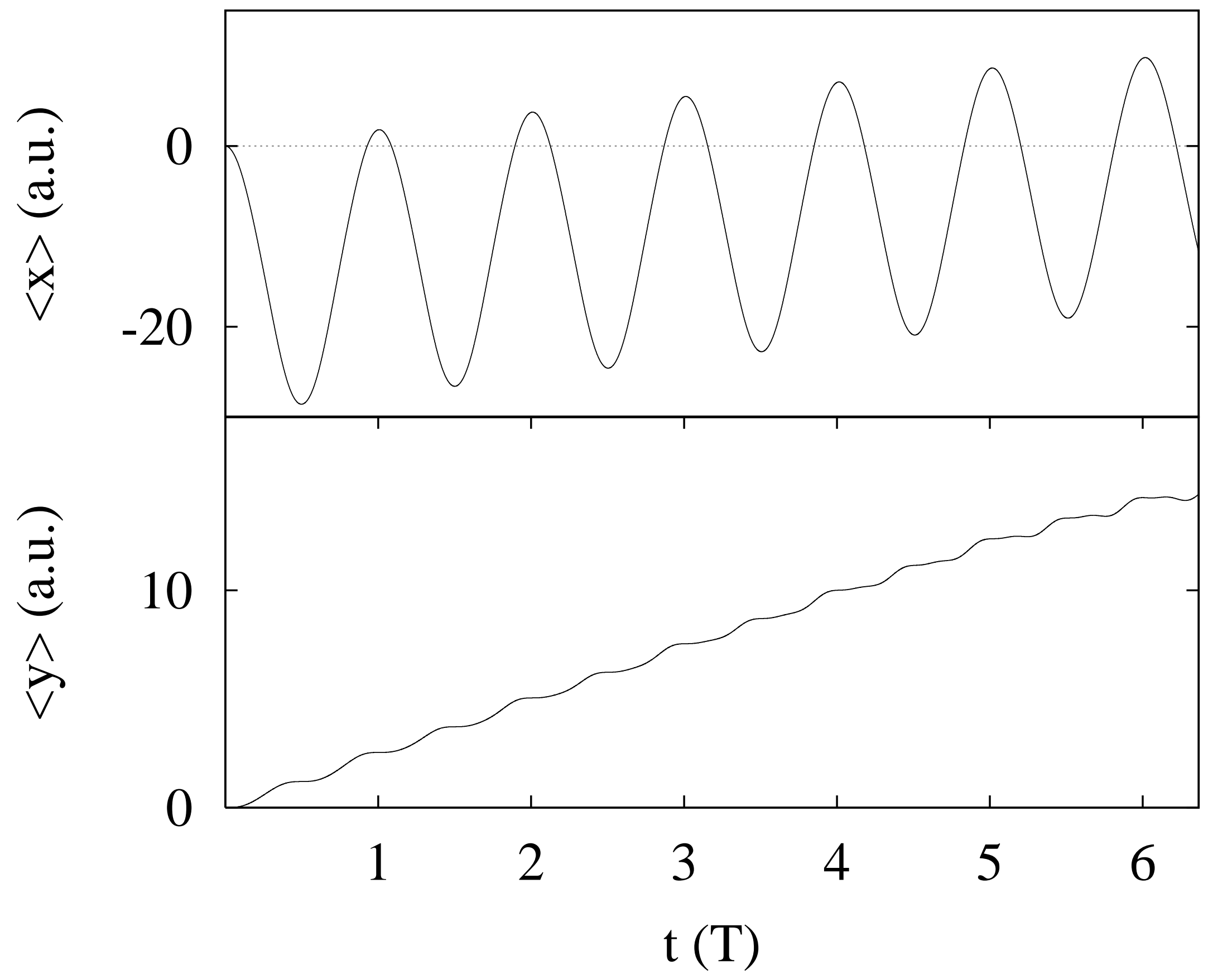




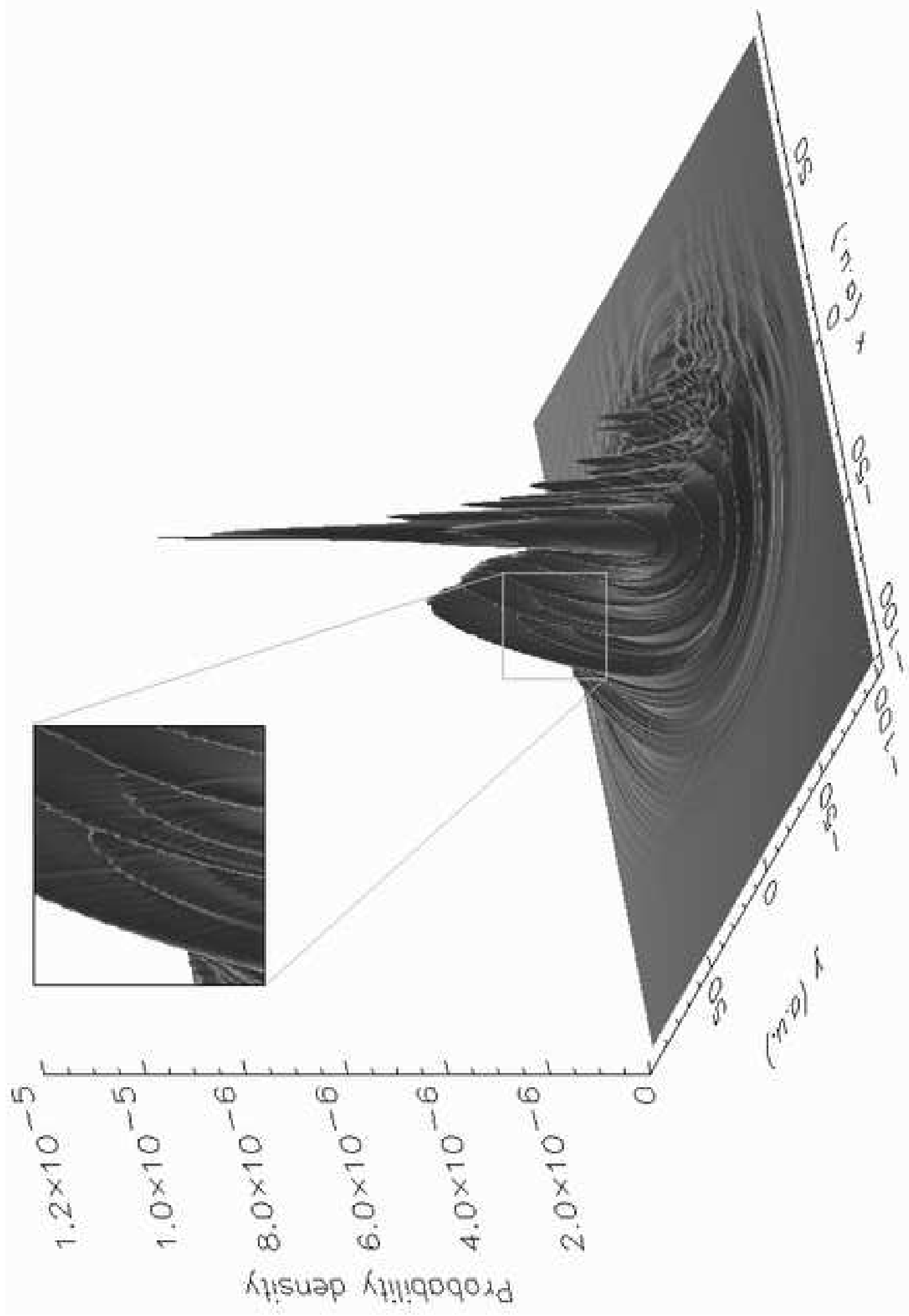




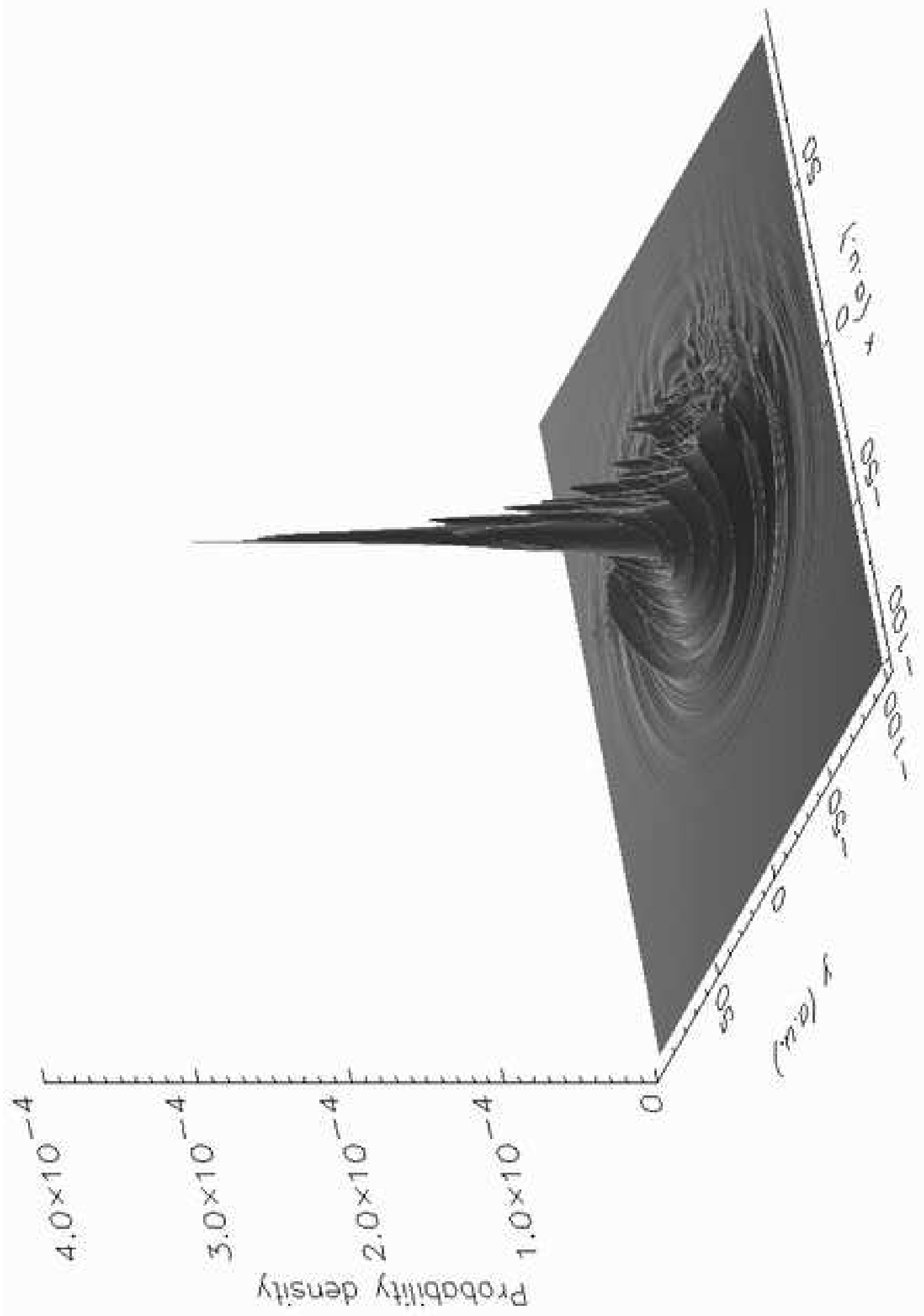



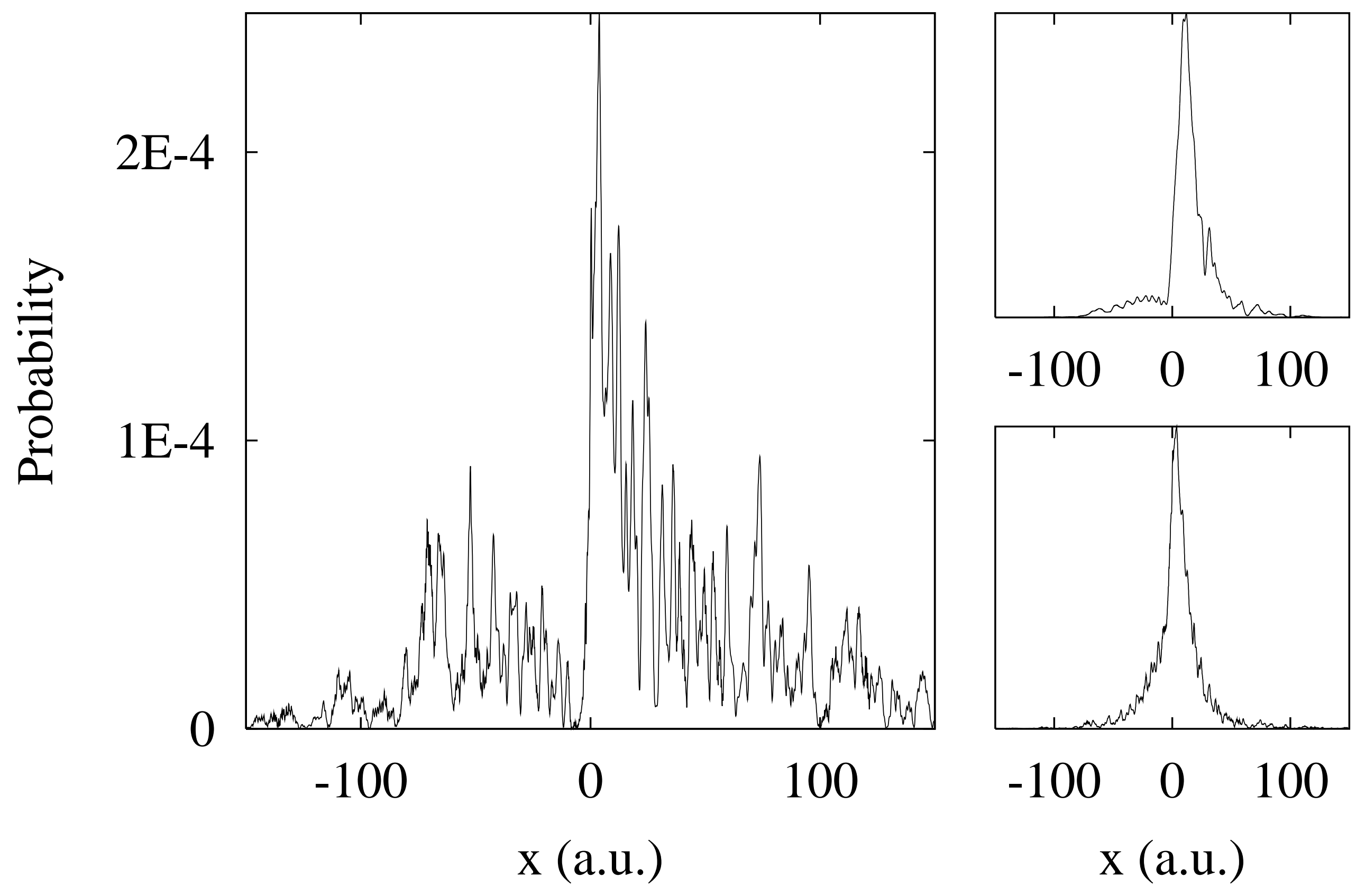

$\begin{array}{lll}-100 & 0 & 100\end{array}$

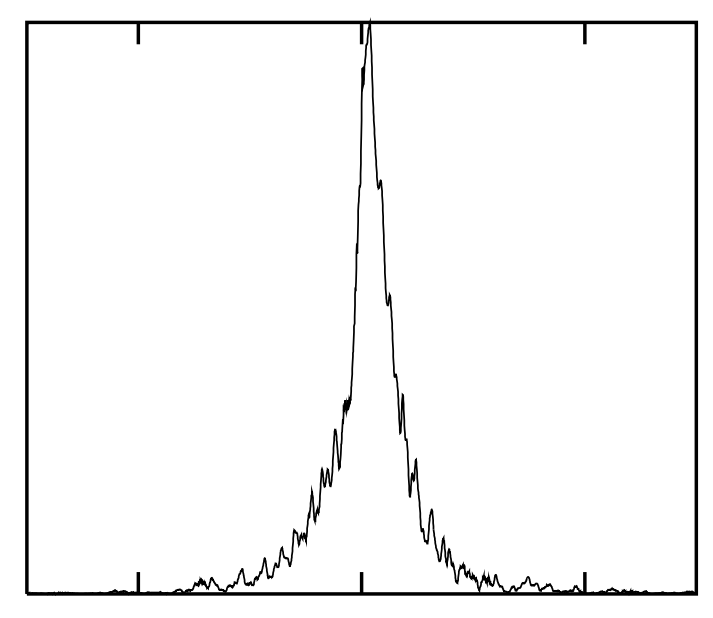

$\begin{array}{lll}-100 & 0 & 100\end{array}$ x (a.u.) 